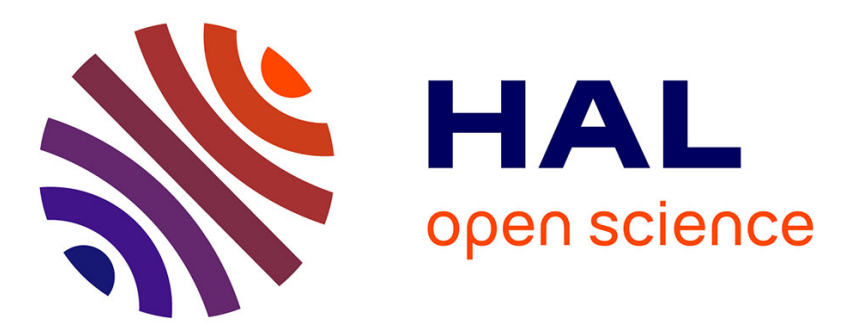

\title{
Enhanced photoemission from laser-excited plasmonic nano-objects in periodic arrays
}

N Fedorov, G. Geoffroy, G. Duchateau, L Štolcová, J Proška, F Novotný, M Domonkos, H. Jouin, P. Martin, Michèle Raynaud

\section{- To cite this version:}

N Fedorov, G. Geoffroy, G. Duchateau, L Štolcová, J Proška, et al.. Enhanced photoemission from laser-excited plasmonic nano-objects in periodic arrays. Journal of Physics: Condensed Matter, 2016, 28 (31), pp.315301. 10.1088/0953-8984/28/31/315301 . hal-01949711

HAL Id: hal-01949711

https://hal.science/hal-01949711

Submitted on 26 Dec 2020

HAL is a multi-disciplinary open access archive for the deposit and dissemination of scientific research documents, whether they are published or not. The documents may come from teaching and research institutions in France or abroad, or from public or private research centers.
L'archive ouverte pluridisciplinaire HAL, est destinée au dépôt et à la diffusion de documents scientifiques de niveau recherche, publiés ou non, émanant des établissements d'enseignement et de recherche français ou étrangers, des laboratoires publics ou privés. 
Enhanced photoemission from laser-excited plasmonic nano-objects in periodic arrays

This content has been downloaded from IOPscience. Please scroll down to see the full text.

2016 J. Phys.: Condens. Matter 28315301

(http://iopscience.iop.org/0953-8984/28/31/315301)

View the table of contents for this issue, or go to the journal homepage for more

Download details:

IP Address: 129.104.29.1

This content was downloaded on 15/06/2016 at 07:40

Please note that terms and conditions apply. 


\title{
Enhanced photoemission from laser-excited plasmonic nano-objects in periodic arrays
}

\author{
N Fedorov ${ }^{1}$, G Geoffroy ${ }^{1}$, G Duchateau ${ }^{1}$, L Štolcová2 ${ }^{2}$ J Proška ${ }^{2}$, \\ F Novotný ${ }^{2,3}$, M Domonkos ${ }^{4}$, H Jouin ${ }^{1}$, P Martin $^{1}$ and M Raynaud ${ }^{5}$ \\ ${ }^{1}$ Université de Bordeaux-CNRS-CEA, Centre Lasers Intenses et Applications, UMR 5107, \\ 351 Cours de la Libération, 33405 Talence, France \\ 2 FNSPE, Czech Technical University in Prague, Brehova 7, CR-11519 Prague, Czech Republic \\ 3 Dept. Surg., University of Ostrava, University Hospital Ostrava, Ostrava, Czech Republic \\ ${ }^{4}$ Institute of Physics v.v.i. ASCR, Cukrovarnicka 10, CR-11519 Prague, Czech Republic \\ 5 Laboratoire des Solides Irradiés, Ecole Polytechnique, CEA, CNRS, Université Paris-Saclay, \\ 91128 Palaiseau cedex, France
}

E-mail: michele.raynaud-brun@polytechnique.edu

Received 23 February 2016, revised 2 May 2016

Accepted for publication 13 May 2016

Published 14 June 2016

\begin{abstract}
The process of photoelectron emission from gold surfaces covered with nano-objects that are organized in the form of a periodic array is addressed in the short laser pulse regime $(\leqslant 50$ fs) at moderate intensities $\sim 10^{10} \mathrm{~W} \mathrm{~cm}^{-2}$ and for various laser wavelengths. The emission spectrum from a gold single crystal measured under the same conditions is used for reference. The comparison of the photo-emission yield and the energy of the ejected electrons with their counterparts from the (more simple) reference system shows that the periodic conditions imposed on the target surface drastically enhance both quantities. In addition to the standard mechanism of Coulomb explosion, a second mechanism comes into play, driven by surface plasmon excitation. This can be clearly demonstrated by varying the laser wavelength. This interpretation of the experimental data is supported by predictions from model calculations that account both for the primary quantum electron emission and for the subsequent surfaceplasmon-driven acceleration in the vacuum. Despite the fact that the incident laser intensity is as low as $\sim 5 \times 10^{10} \mathrm{~W} \mathrm{~cm}^{-2}$, such a structured target permits generating electrons with energies as high as $300 \mathrm{eV}$. Experiments with two incident laser beams of different wavelengths with an adjustable delay, have also been carried out. The results show that there exist various channels for the decay of the photo-emission signal, depending on the target type. These observations are shedding light on the various relaxation mechanisms that take place on different timescales.
\end{abstract}

Keywords: photoemission, laser excitation, surface plasmon, plasmonics

(Some figures may appear in colour only in the online journal)

\section{Introduction}

Over the last decade, metallic nano-particles have attracted tremendous interest. This is due to their unique optical properties which hold the promise of opening the way to controlling light on the nanometer length scale and femtosecond time scale $[1,2]$. In particular, the excitation of non-propagating surface plasmons (SP) in metallic nano-particules is of great interest [1, 3-5] as it has been observed to lead to a significant enhancement of the absorption ( viz. more than 50\%) when the excitation is triggered by an ultra-fast laser pulse. This may give rise to an enhancement of the local intensity and a sub-wavelength confinement of the optical field, due to the finite size of the objects [6-8]. This in turn leads to intense field dynamics whereby the multi-photon emission can be strongly enhanced [9-13]. The SP evanescent field penetrates the vacuum over a very short distance, such that a strong acceleration of the electrons may be observed [14-17] due 
to the ponderomotive force within the local, induced field. Such a mechanism was first reported in grating targets where propagative surface plasmons might/can be excited [18-27]. Electrons with energies as high as tens of $\mathrm{eV}$ could be obtained for laser intensities as low as $\sim 10^{10} \mathrm{~W} \mathrm{~cm}^{-2}$. With the recent development of CEP-stabilized few-cycle laser pulses, it has become possible to obtain higher energy cut-offs (hundreds of $\mathrm{eV}$ ) in the photoelectron spectra of metallic nanoparticles $[15,28,29]$. This point is of considerable interest for the processes and devices that exploit hot-electron emission, e.g. in photo-catalysis, photovoltaïc devices and optoelectronics.

For a single nanoparticle, the SP strongly depends on the particle shape [30], on its dielectric properties, and on the nature of the surrounding medium. When-as in most experimental situations - a set of nanoparticles is considered, the SP is further influenced by the electromagnetic interactions between the particles [31]. For example, the use of periodic arrays has emerged recently as a promising tool for plasmonic and photonic applications in optics [32-35]. In order to improve the electron sources in terms of emissivity, energy and beam spread, one can also take advantage of metallic nanoparticles organized within a network to enhance the overall plasmon resonance of the system [16, 17, 36-39]. Actually the precise mechanism for the energy transfer and relaxation that leads to the drastic enhancement of the photo-emission properties during the interaction of the laser with the nano-particle array is still not well understood in the short-pulse regime. There can thus be no doubt that in order to optimize new highemission devices, an improved knowledge of the relevant mechanism of electron transfer and relaxation is required.

In the present work we have studied the photoelectron emission obtained by short-pulse ( $\sim 25 \mathrm{fs})$ laser excitation of a regular two-dimensional array of gold-coated nano-scale objects $(\mathrm{N}-\mathrm{O})$ within a range of moderate laser intensities $\sim 10^{10} \mathrm{~W} \mathrm{~cm}{ }^{-2}$. The goal is to obtain a target with an efficient plasmon structure and to understand the role played by the excitation, whose wavelength matches that of the laser. The radius of the nano-scale objects is about $215 \mathrm{~nm}$ and from now on we will call these targets $\mathrm{N}-\mathrm{O}$ array targets. Their properties have been studied as a function of the laser wavelength (which was varied between 800 and $266 \mathrm{~nm}$ ), with laser intensities ranging from $10^{8}$ to $5 \times 10^{10} \mathrm{~W} \mathrm{~cm}^{-2}$. The electron relaxation and heat diffusion of the $\mathrm{N}-\mathrm{O}$ array target have been also investigated in this work as it might be important for applications in electron tunneling devices. It is well-known that a laser photon cannot couple to the surface plasmon wave of a flat surface (unless in some special excitation geometries such as the one reported by Kretschmann [40, 41]). This is the reason why we will compare the results on the $\mathrm{N}-\mathrm{O}$ array target with those obtained with a gold single-crystal sample, which should be devoid of surface plasmon effects. This gold single crystal will thus be used as a reference sample.

In our study we observe a large increase in both the photoemission yield and the photoelectron energy of the N-O array targets in comparison with the gold single crystal. Due to the presence of the 2D array of nano-particles on the surface, the former exhibits a periodic roughness which triggers the excitation of a propagative surface plasmon. Consequently, the photoemission yield depends on the laser incidence. This situation is quite different from those encountered in previous studies on isolated nano-particles that were deposited onto a support. Two different interaction regimes are analyzed here: at a laser wavelength of $266 \mathrm{~nm}$ (which is of the order of the size of the $\mathrm{N}-\mathrm{O}$ ), and at $800 \mathrm{~nm}$ (with the aim of investigating the dipolar regime wherein higher local-field enhancements are expected). In particular, it is shown that the use of N-O array targets permits accelerating electrons to kinetic energies which are as high as roughly $300 \mathrm{eV}$, even when only moderate laser intensities (of the order of $5 \times 10^{10} \mathrm{~W} \mathrm{~cm}^{-2}$ ) are applied. Two-color pump-pump experiments whereby the laser pulse duration is much shorter than the time scale on which the energies are redistributed by the electron-electron and electron-lattice interactions are now commonly used techniques. They are efficient to study the energy redistribution during the laser-solid interaction [42, 43]. Such experiments have also been performed here in order to investigate the differences between the electronic relaxation dynamics in the $\mathrm{N}-\mathrm{O}$ array targets and in the gold single crystal. For all experimental observations, we will provide qualitative interpretations that shed light on the underlying physical processes that may be taking place.

The paper is organized as follows: first, the N-O array target synthesis and the framework of the photoemission experiments are presented in section 2 . Next, the experimental results are detailed and discussed for both types of targets, i.e. the single-crystal and the N-O array targets. This happens in section 3, where we first investigate the possibility of exciting a propagative surface plasmon on the structured target (section 3.1) with a laser. Then we compare the electron emission obtained at low laser intensities from the gold single-crystal sample (section 3.2.1) and the $\mathrm{N}-\mathrm{O}$ array target (section 3.2.2) with those derived from a scenario wherein the mechanism for electron acceleration is based on space charge effects, i.e. Coulomb repulsion. In order to back our interpretations of the experimental data on the wavelength dependence of the photo-emission process, we present in section 3.3 predictions obtained from model calculations that account for both the primary quantum electron emission and the subsequent surface plasmon-driven acceleration in the vacuum. The response observed for the two types of targets in the regime of relatively high laser intensity is reported in section 3.4. Finally, we also present pump-pump experiments in section 3.5, as they offer the opportunity to highlight the differences between the electronic relaxation dynamics in the two sample types in a novel way. Further consequences for possible applications are given in the concluding section 4 .

\section{Experimental configuration}

\subsection{Targets preparation}

A monolayer of polystyrene microspheres of $940 \mathrm{~nm}$ diameter (microParticles, GmbH, Berlin, Germany) in the form of an hexagonal lattice with close-packed order was prepared by self-assembly at the water/air interface (following a technique described in [44]). It was subsequently deposited onto 


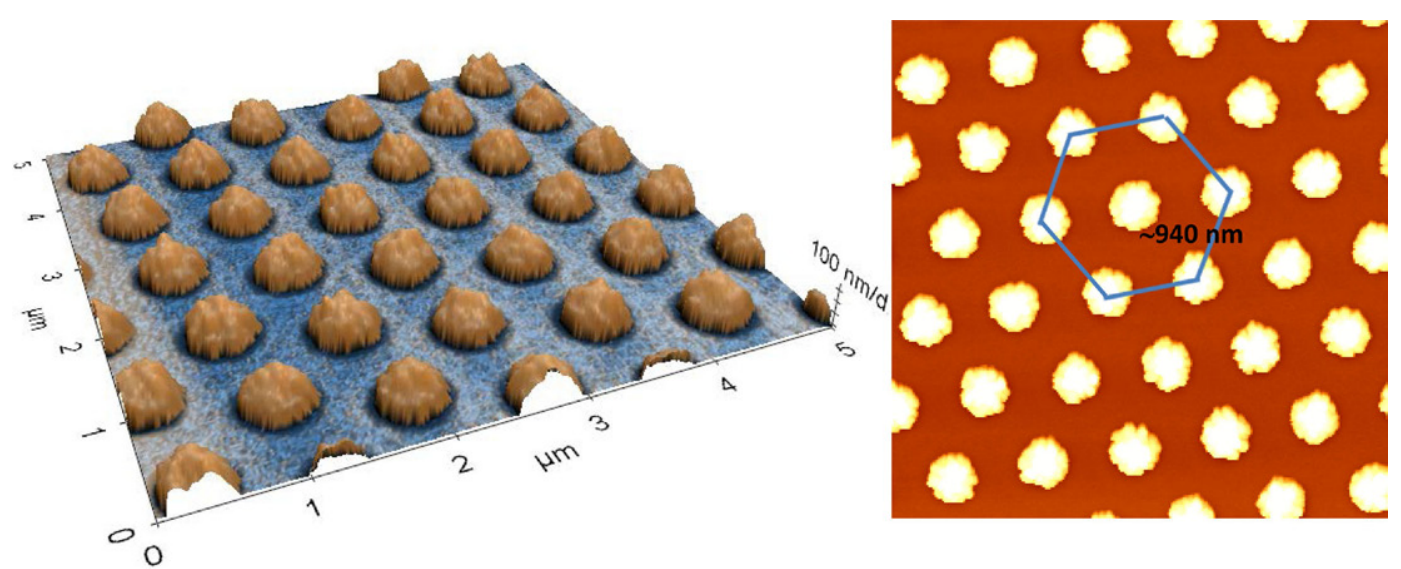

Figure 1. Image of the N-O array target. The objects have a radius of about $215 \mathrm{~nm}$ and are arranged in the form of a hexagonal honeycomb lattice with a lattice parameter of $\sim 940 \mathrm{~nm}$.

a cleaned glass substrate. After drying, the size of the microspheres was decreased by dry etching in a reactive ion etching plasma system (Phantom III/Phantom LT RIE System, Trion Technology) operated in a capacitative-coupling mode for $135 \mathrm{~s}$. The total gas pressure was 90 mTorr, the RF power was $100 \mathrm{~W}$, and the $\mathrm{O}_{2}$ flow rate was $50 \mathrm{sccm}$. A gold layer of $60 \mathrm{~nm}$ thickness was laid over the etched polystyrene spheres by magnetron sputtering in a high-resolution sputter coater (Cressington 208HR). The gold-coated nano-scale objects obtained by this procedure had maintained their periodicity, preserving the lattice parameter of the close-packed spheres and their main radius of $215 \mathrm{~nm}$ (see figure 1).

This sample will be referred to hereafter as the N-O array target. In order to compare the influence of the finite size of the objects on the dynamics of the electronic relaxation, a second N-O array target has been prepared in the same way. It consists of nano-objects of a smaller size (radius $<100 \mathrm{~nm}$ ) and has been used in the pump-pump experiments. The latter sample will be referred to as the NO-100 array target.

\subsection{Photoemission measurements}

The experiments have been carried out by using the $1 \mathrm{kHz}$ Ti:Sa Aurore laser system at CELIA (Centre Laser Intense et Applications), providing $8 \mathrm{~mJ}, 25 \mathrm{fs}$ laser pulses at $800 \mathrm{~nm}$. The output frequency is doubled and tripled using a BBO crystal to generate $266 \mathrm{~nm}$ light pulses. The duration of the $266 \mathrm{~nm}$ pulses has been estimated to be 50 fs. The photoemitted spectra were recorded at the three laser wavelengths for laser intensities ranging from 0.3 to $50 \mathrm{GW} \mathrm{cm}^{-2}$. These values have been deduced from a calorimetric measurement of the pulse energy (with shot-to-shot fluctuations of 5\%) and from the autocorrelation determination of the pulse duration (with a 5\% uncertainty). The laser beam is $p$-polarized and is impinging onto the target without focalization (the focal-spot diameter on the target equals $\sim 1.5 \mathrm{~mm}$ ).

The sample is mounted within a magnetically shielded, high-vacuum $\left(\sim 10^{-9}\right.$ mbar $)$ chamber and can be rotated around its vertical axis to change the incidence angle of the laser beam with respect to its surface. The kinetic-energy distribution of the electrons is analyzed with a hemispherical detector CLAM IV. The direction of detection was perpendicular to the sample surface. When the electron emission is relatively large, the detection saturates at about 3500 counts $\mathrm{s}^{-1}$ due to electron losses during their transport in the CLAM. This is of minor importance in the following analysis since we are mainly interested in the high-energy part of the photoelectron spectra. We determined the energy resolution of the electron spectrometer to be $0.1 \mathrm{meV}$ for an electron with a kinetic energy of $50 \mathrm{eV}$.

The time-resolved two-color photoemission experiments have been performed using the fundamental of the Ti-Sa laser system $(800 \mathrm{~nm}, 25 \mathrm{fs})$ as pump and the tripled-energy beam ( $266 \mathrm{~nm}, 50 \mathrm{fs})$ as the second pump. The two laser pulses are delayed with respect to each other by means of an opticalmirror device on a moving plate with a spatial resolution of $1 \mu \mathrm{m}$ (corresponding to $6.6 \mathrm{fs}$ ). The two pulses have identical focal spot diameters and they are both $p$-polarized. Note that the noise in the spectra from N-O array targets is higher than that in the spectra from the single crystal, due to the roughness of the surface and the laser incidence angle of $45^{\circ}$. It is presumably due to some fluctuations in the spatial (and therefore temporal) overlapping of the two laser beams. Photoelectron spectra have also been recorded before and after the two-color pump-pump scans to ensure that the sample properties had not been altered during the scans.

All the measurements presented here have been collected well below the damage threshold of the targets, and at room temperature (close to $300 \mathrm{~K}$ ).

\section{Results and discussion}

\subsection{Angular dependence of the photoemission yield}

It is well-known that the surface plasmon excitation strongly influences the photoemission from metallic nano-particles $[13,16,17]$ and metallic gratings [18-27]. Since the target is covered with a lattice of quasi single-sized objects, the latter can act as a structured surface, in analogy with the situation encountered in a metal grating. It can therefore provide the incremental momentum required to fulfill the condition of momentum conservation in the excitation of the 


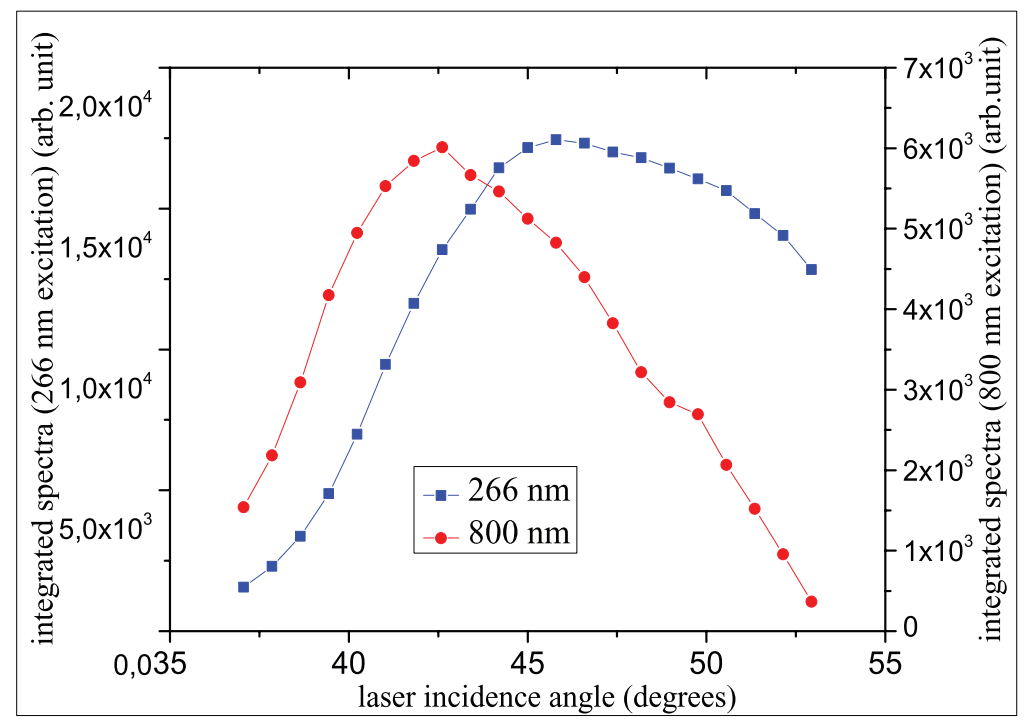

Figure 2. Integrated photoemission signal of the $\mathrm{N}-\mathrm{O}$ array target as a function of the laser incidence angle (measured with respect to the normal to the surface) at $800 \mathrm{~nm}, 25 \mathrm{fs}$ (red filled circles) and $266 \mathrm{~nm}, 50 \mathrm{fs}$ (blue filled squares) at $\sim 0.3 \mathrm{GW}^{-2}$.

surface plasmon by the laser. In this case the photoemission is expected to reach a maximum for some optimal laser angle of incidence. This stands in marked contrast with the situation encountered in the case of a single nano-particle, and this fact has in return motivated the study of the angular dependence of the photoemission reported hereafter.

Figure 2 displays the integrated photoemission signal as a function of the angle of incidence of the laser beam for the N-O array target, at two laser wavelengths (of 800 and $266 \mathrm{~nm}$ ) and a laser intensity of $\sim 0.3 \mathrm{GW} \mathrm{cm}^{-2}$. At $800 \mathrm{~nm}$ $(h \nu=1.55 \mathrm{eV})$, the photoemission signal reaches a maximum around the laser beam incidence angle of $42.5^{\circ}$ and the signal width is relatively large $\left(\sim 10^{\circ}\right.$ at half the maximum). At $266 \mathrm{~nm}(h \nu=4.65 \mathrm{eV})$ the yield of the electron emission is higher than at $800 \mathrm{~nm}$ since the order of the multi-photon process is lower. The maximum of the photoemission signal is shifted towards an incident angle of $47^{\circ}$. Since we are not in the dipolar regime $\left(\lambda_{\mathrm{L}}=266 \mathrm{~nm}\right.$, which is of the the order of the radius $r_{0} \simeq 215 \mathrm{~nm}$ of the objects), the resonance is larger ( $\sim 15^{\circ}$ at half the maximum) and is not so well defined. At resonance, one expects that the surface plasmon excitations will produce a locally amplified electric field on the target surface, just like in the case of a grating surface. Also here this field is influenced by the roughness of the gold surface, the shape of the individual nano-objects, their mutual interactions, and their interactions with the substrate. Note also that we do not observe any dependence of the emission on the laser polarization. This can be explained as a consequence of the quasispherical shape of the nano-objects.

It has to be emphasized that changing the incident laser wavelength, $\lambda_{\mathrm{L}}$, modifies the laser interaction regime. This is bound to translate into an effect on both the collective and the local effects. As a matter of fact, at $\lambda_{\mathrm{L}}=800 \mathrm{~nm}$, we have $r_{0} / \lambda_{\mathrm{L}} \ll 1$ such that we are in the dipolar regime. In this case, a high local-field amplification is expected (see section 3.2). This may induce a ponderomotive acceleration of the photoemitted electrons [27]. On the contrary for $\lambda_{\mathrm{L}}=266 \mathrm{~nm}$, $r_{0} / \lambda_{\mathrm{L}} \sim 1$ such that retardation effects are no longer negligible. Moreover, $h \nu$ is above the threshold for the inter-band transition in gold (2.3 eV [45]). This may lead to further damping of the surface plasmon resonances and a lower local-field amplification. This effect is not clearly seen in figure 2 as it is blunted by the enhancement of the photoemission probability at $266 \mathrm{~nm}$ due to the lower multi-photon order.

No angular dependence of the photoelectron emission yield is observed for the gold single-crystal target under the same conditions. This is as expected, as it is just impossible to excite surface plasmons in this case. The fact that we find an angular dependence in the photoemission from the N-O array target is evidence for the presence of a propagative surface plasmon excited by the laser. Therefore in the following, all spectra reported at $800 \mathrm{~nm}$ and $266 \mathrm{~nm}$, have been recorded at an angle of incidence of $45^{\circ}$, both for the $\mathrm{N}-\mathrm{O}$ array target and for the single-crystal target. At this incidence angle the conditions for the excitation of a surface plasmon are fulfilled.

\subsection{Photoemission spectra at low laser intensities}

3.2.1. Gold single-crystal target. We start by analyzing the case of the gold single crystal as it must serve for further reference. First we investigate the influence of the pulse duration in order to determine whether space charge effects may be significant or otherwise. With this goal in mind, the photo-emission spectra at a wavelength of $800 \mathrm{~nm}$ are plotted in figure 3 for various laser intensities and two values of the pulse duration ( $25 \mathrm{fs}$ and $220 \mathrm{fs}$ ). Our procedure is such that the pulse duration is changed, resulting in energy variations from $0.5 \mu \mathrm{J}$ ( $\sim 0.65 \mathrm{GW} \mathrm{cm}^{-2}$ for $\left.25 \mathrm{fs}\right)$ to $3.5 \mu \mathrm{J}\left(\sim 4.57 \mathrm{GW} \mathrm{cm}^{-2}\right.$ for $25 \mathrm{fs})$. It transpires that within this range of laser energies, increasing the pulse duration by a factor $\sim 8$ while keeping the pulse energy constant does not affect the position of the energy for threshold emission nor the shape of the spectra. We thus conclude that space charge effects are negligible under these conditions [46]. The standard photoelectron spectrum is 

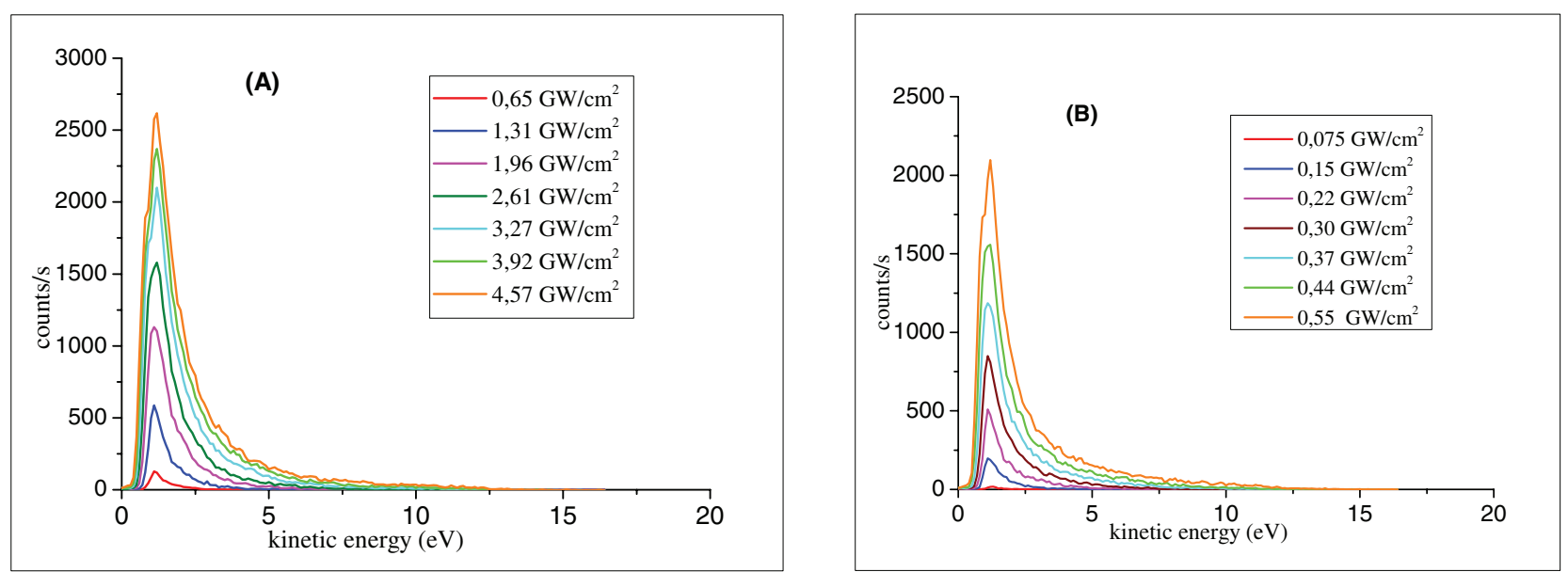

Figure 3. Spectra of photo-emitted electrons from a gold single crystal irradiated with a $800 \mathrm{~nm}$ laser pulse for increasing laser intensities: (A) with a pulse duration of $25 \mathrm{fs}$; (B) with a pulse duration of $220 \mathrm{fs}$.
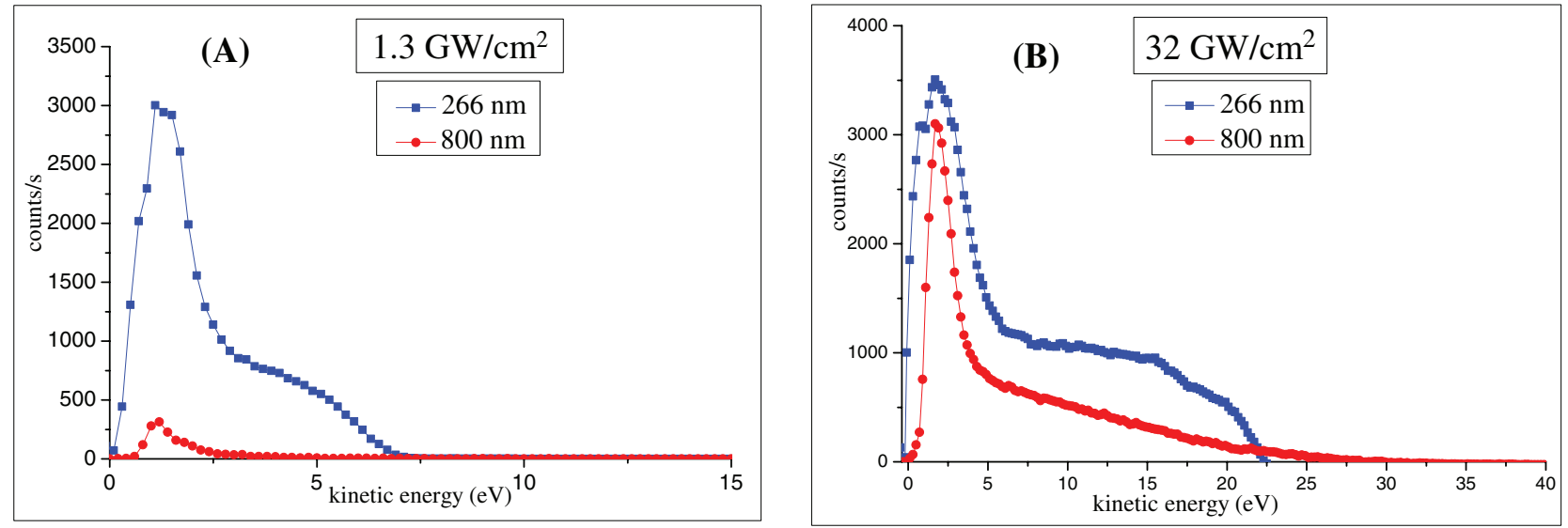

Figure 4. (A) Spectra of photo-emitted electrons from a gold single crystal irradiated with a $800 \mathrm{~nm}$ (red filled circles), and $266 \mathrm{~nm}$ (blue filled squares) laser pulse at $1.3 \mathrm{GW} \mathrm{cm}^{-2}$. (B) the same for an intensity of $32 \mathrm{GW} \mathrm{cm}^{-2}$ (the detector saturation is about 3500 counts $\mathrm{s}^{-1}$ due to electron losses during their transport in the CLAM (see also text)).

recovered from this target, which presents quite a low photoemission yield. This is the result of a multi-photon absorption process that occurs at this laser wavelength. Using the law of conservation of energy, the position of the peak at low kinetic energy can be used to estimate the work function around $5 \mathrm{eV}$. It is in agreement with previously reported standard values for gold [47]. Note that this experiment is not designed to study the low-energy electrons and extract an accurate value of the metal work function in fine detail. The purpose is here rather to focus the attention onto the high-energy electrons.

The influence of the laser wavelength is illustrated by figure 4 which shows photo-emission spectra for the shortest available pulse duration ( $25 \mathrm{fs}$ ) at both $800 \mathrm{~nm}$ and $266 \mathrm{~nm}$ for two intensities: (A) $1.3 \mathrm{GW} \mathrm{cm}^{-2}$ and (B) $32 \mathrm{GW} \mathrm{cm}^{-2}$. We remind that the detection saturates around 3500 counts $\mathrm{s}^{-1}$. When we decrease the laser wavelength from 800 to $266 \mathrm{~nm}$ keeping a low laser intensity as reported in figure 4(A), we observe that the shorter the laser wavelength is: (a) the higher the density of the electrons emitted is, (b) and the higher the maximum of the electron kinetic energy is, while (c) the energy position of the main peak is slightly shifted to higher energies. This last point will be discussed in more details in the modeling support section. When the laser intensity is increased from $1.3 \mathrm{GW} \mathrm{cm} \mathrm{cm}^{-2}$ to $32 \mathrm{GW} \mathrm{cm} \mathrm{cm}^{-2}$ a significant modification of the spectral shape takes place in its highenergy part. Several physical mechanisms may explain this behavior. Firstly, the shorter the wavelength is, the smaller the order of the multi-photon process will be. Thus the probability of emission is increased and more electrons are produced, as observed in the experiment. Therefore, charge repulsion may take place inside the electron bunch under these conditions. This induces additional electron deceleration and acceleration [46]. Secondly, the production of a large electron density entails a larger number of electron collisions, and this leads to a faster relaxation of the electron gas. This could also be responsible for the particular shape of the spectra, which looks rather similar to a Fermi-Dirac distribution. The latter accounts for a non-independent electron gas that has reached equilibrium by relaxing via electron-electron collisions. Furthermore, at low electronic temperature, the chemical potential is of the order of the Fermi energy $E_{\mathrm{F}}$ which varies as $n_{\mathrm{e}}^{2 / 3}$ where $n_{\mathrm{e}}$ is the produced electron density. The maximum value of the kinetic energy of the electrons may thus be related to the relaxation processes. Moreover, for decreasing wavelengths, the slope 


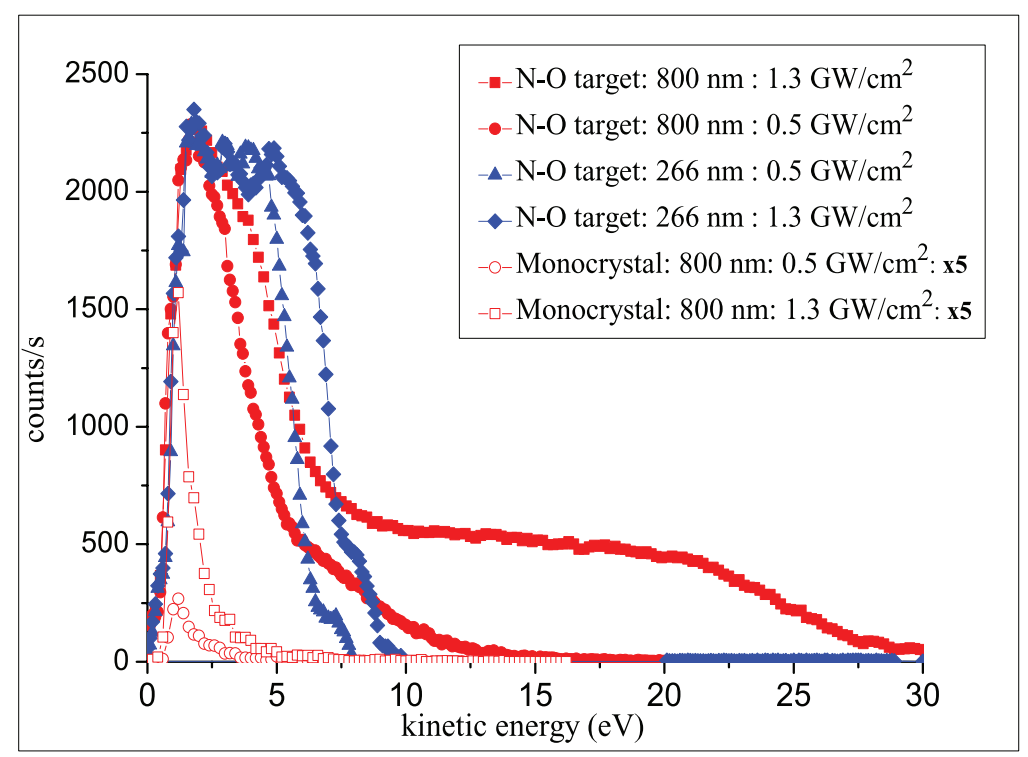

Figure 5. Spectra of the photo-emitted electrons from the N-O array target irradiated with a $800 \mathrm{~nm}, 25 \mathrm{fs}$ laser pulse with a laser intensity of $0.5 \mathrm{GW} \mathrm{cm}^{-2}$ (red filled circles) and of $1.3 \mathrm{GW} \mathrm{cm}^{-2}$ (red filled squares) and with a $266 \mathrm{~nm}, 50$ fs laser pulse with a laser intensity of $0.5 \mathrm{GW} \mathrm{cm}^{-2}$ (blue filled triangles) and of $1.3 \mathrm{GW} \mathrm{cm}^{-2}$ (blue filled diamonds). For comparison we display also the spectra of the electrons photo-emitted by a gold single crystal (multiplied by 5) irradiated with a $800 \mathrm{~nm}, 25 \mathrm{fs}$ laser pulse at $0.5 \mathrm{GW} \mathrm{cm}^{-2}$ (red circles) and $1.3 \mathrm{GW} \mathrm{cm}^{-2}$ (red squares).

of the spectra increases in its high-energy part. When it can be assumed that the electron gas has reached equilibrium, the slope is directly related to the electron temperature: the higher the temperature, the smaller the slope of the distribution (around the Fermi energy). The decrease in the slope of the distribution with increasing laser wavelengths suggests thus an increase in the electron temperature. This is in agreement with standard models of laser electron heating in metals [48]. Indeed, the transition rate for photon absorption by free electrons increases as a function of the wavelength.

To conclude this analysis, the photoemission behavior of the gold single-crystal can be rather well understood by considering both space charge effects and laser heating of the electrons. The relative contributions of these two mechanisms depend both on the laser wavelength and intensity.

3.2.2. N-O target. Let us now consider the case of the N-O array target. The photoelectron spectra are reported in figure 5 at $800 \mathrm{~nm}$ for two laser intensities: $1.3 \mathrm{GW} \mathrm{cm}^{-2}$ and $0.5 \mathrm{GW} \mathrm{cm}^{-2}$. For comparison, the spectrum of the gold single crystal is also displayed after multiplication by a factor of 5 . The most salient feature here is the difference in the photo-emitted current between the two sample types. This current is enhanced by a factor $\sim 50$ in the N-O array target in comparison with the single-crystal target. Moreover, we note the presence in the spectra of electrons with an energy that is higher than can be expected based on the photoelectric balance: up to $\sim 15 \mathrm{eV}$ and $\sim 30 \mathrm{eV}$ at $0.5 \mathrm{GW} \mathrm{cm}^{-2}$ and $1.3 \mathrm{GW} \mathrm{cm}^{-2}$, respectively.

The evolutions observed may be interpreted in terms of two main mechanisms: the ponderomotive acceleration of the electrons in the high-frequency inhomogeneous local field induced by laser excitation of surface plasmons $[18,27]$ and a significant charge repulsion. The latter may take place in the emitted electron bunch.
Let us first discuss the mechanism of ponderomotive electron acceleration. When the electrons are released from a metal that carries a SP excitation, they will be further subjected to the inhomogeneous high-frequency longitudinal SP field in the vacuum. Consequently, the total energy of an emitted electron will be the result of two contributions: the kinetic energy gained after overcoming the metal work function and the ponderomotive potential $U_{\mathrm{p}}=E_{\mathrm{SP}}^{2} / 4 \omega_{0}^{2}$ in the oscillating SP field $E_{\mathrm{SP}}$. Here $\omega_{0}$ is the angular frequency of the laser. This will be correct provided the electrons are given enough time to explore the full SP field gradient. It is worth noting that the SP field amplitude is much higher than the amplitude of the laser field. It is this feature which is responsible for the high electron yields that are obtained when a SP excitation is present. The ponderomotive potential is then high even when the laser intensities are as low as those considered in the present work. The electrons will thus be significantly accelerated [18, 19]. Moreover, a part of the electrons may during their motion bounce back after their initial acceleration in the SP field. This is then due to the change of the sign of the field. The magnitude of the time interval during which the electron is bouncing back is such that it acts as a kind of reset for the initial conditions of the particle. This way the electron can be accelerated once more in the next half-cycle of the high-frequency field $[15,17,49,50]$. This effect further broadens the final energy distribution of the electrons towards higher values.

The electron acceleration observed may also be interpreted as a consequence of the strong charge repulsion that may prevail within the emitted electron bunch. This idea is partly supported by a comparison of the photo-emission spectra shown in figure 6 . Here the pulse duration has been increased by a factor $\sim 8$ from 25 fs to 220 fs while keeping the pulse energy constant. Although no shift of the emission threshold is observed, the cut-off energy of the emitted electrons decreases when one 

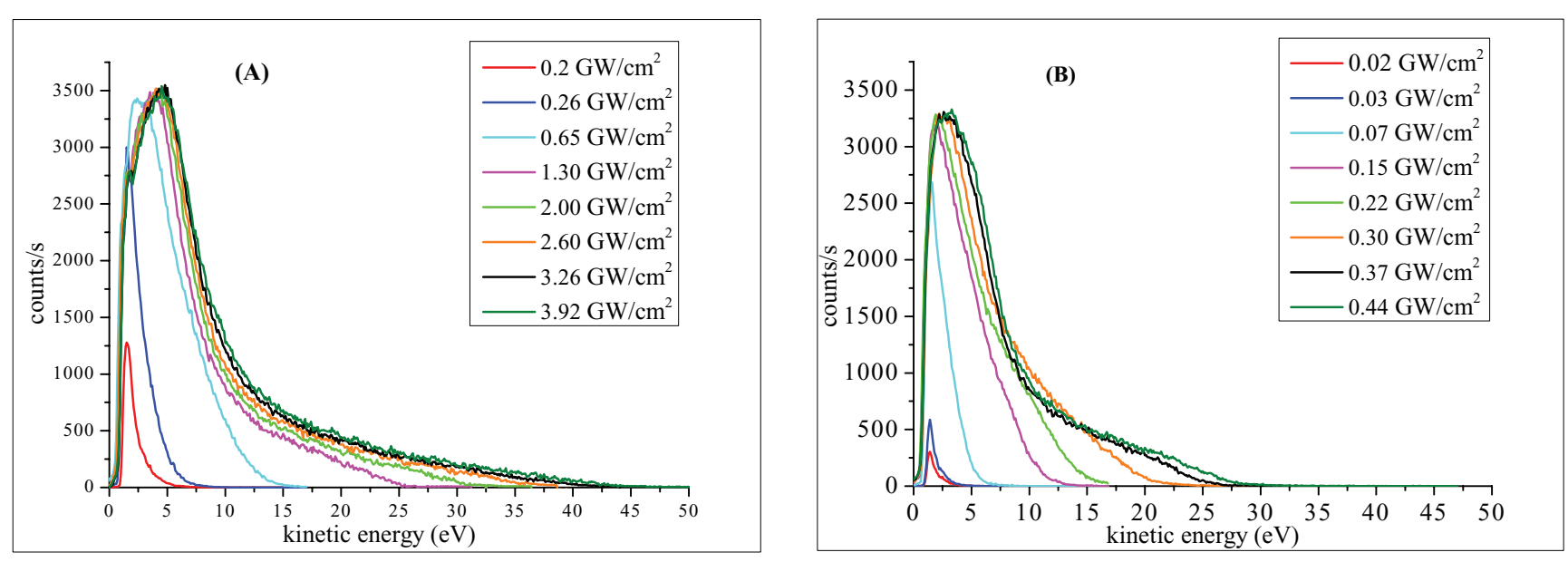

Figure 6. Spectra of photo-emitted electrons from a gold N-O array target irradiated with $800 \mathrm{~nm}$ laser pulses for increasing laser energies: (A) with a pulse duration of $25 \mathrm{fs}$, (B) with a pulse duration of $220 \mathrm{fs}$.

increases the duration of the pulse keeping the pulse energy constant. Indeed, at constant laser energy, longer pulses correspond to lower intensities, and these lead to smaller densities of the ejected electrons. The charge repulsion effects become thus less important when the pulse duration is increased. Note that the ponderomotive acceleration, which varies as $I \lambda^{2}$, differs from the acceleration due to the presence of space charge by its dependence on the duration of the laser pulses. In fact, increasing the laser pulse duration from 25 fs to 220 fs results in a more complete conversion of the ponderomotive energy. The energy gain of the electrons becomes higher at constant laser intensity [18]. This is clearly observed on the spectra shown in figure 6 when one compares the spectra reported at 25 fs for $0.15 \mu \mathrm{J}\left(0.2 \mathrm{GW} \mathrm{cm}^{-2}\right)$ with the spectrum reported at 220 fs for $3 \mu \mathrm{J}\left(0.44 \mathrm{GW} \mathrm{cm}^{-2}\right)$.

With the N-O array target (see figure 5), the maximal kinetic energy of the electrons obtained with laser pulses of a wavelength of $266 \mathrm{~nm}$ is less than that obtained with pulses of a wavelength of $800 \mathrm{~nm}$. This behavior is opposite to the behaviour previously reported for the single-crystal sample (see figure 4). It is consistent with the $I \lambda^{2}$ wavelength dependence expected for the ponderomotive acceleration of electrons in vacuum due to the local induced SP field. And it is further reinforced by the fact that at this wavelength we are well above the threshold for inter-band transitions in gold [45] $(2.3 \mathrm{eV})$. Thus, the surface plasmon is damped and the associated local field reduced. At $266 \mathrm{~nm}$, another effect can be observed: the main peak, centered at an energy of roughly $3 \mathrm{eV}$, may split into two parts at its maximum. In fact, as previously mentioned, for this wavelength the probability of photoemission is higher (two-photon process). Hence, a larger amount of photo-emitted electrons is released during the rather short duration of the laser pulse (50 fs). This may lead to charge repulsion within the emitted electron bunch and subsequently split the bunch. This in turn will further accelerate the electrons [46]. Increasing the laser intensity from 0.5 to $1.3 \mathrm{GW}$ $\mathrm{cm}^{-2}$ increases the produced electron density, thus enhancing the Coulomb repulsion and the splitting of the electron bunch. Such an enhancement is indeed observed in the experimental spectra. It should be noted however, that the acceleration due to the Coulomb repulsion is smaller at $266 \mathrm{~nm}$ than at $800 \mathrm{~nm}$, which shows that that the ponderomotive electron acceleration is the dominant mechanism for producing high-energy electrons.

Based on the previous observations and analysis it is possible to propose the following as a likely physical scenario for the electron dynamics during the emission process when the SP is excited in the N-O array target. First of all, from the fact that the presence of a SP excitation enhances the electron emission, it can be concluded that both the Coulomb and ponderomotive mechanisms (including half-cycle re-acceleration) play a role. The electrons are first emitted as a dense bunch wherein electron-electron collisions and Coulomb repulsion take place. During this step, the electron gas may partially relax, giving rise to a distribution that has reached equilibrium for the high-energy electrons, which are the ones that have undergone the largest number of collisions. At lower energies, the distribution broadens by a few eV due to the Coulomb repulsion. While the bunch expands, this Coulomb repulsion vanishes. The electrons then become independent one from another, and in a second step they can be further accelerated through the ponderomotive effect.

To conclude this part, we have shown that when the surface plasmon is laser excited (i) the N-O array targets have a higher photoemission yield than the gold single crystal while (ii) the energy of the electrons emitted is higher. This behavior can be qualitatively interpreted by considering both space charge effects and electron ponderomotive acceleration within the local induced surface plasmon field in vacuum, whereby the relative contributions depend on both the laser wavelength and intensity.

\subsection{Modeling support}

The aim of this section is to verify if the ponderomotive effect can contribute to the observed wavelength dependence of the high-energy part of photoemission spectra when the surface plasmon is excited. This part of the spectrum is different in the single-crystal sample and in the N-O array target on which the surface plasmon is excited. The problem addressed here is 

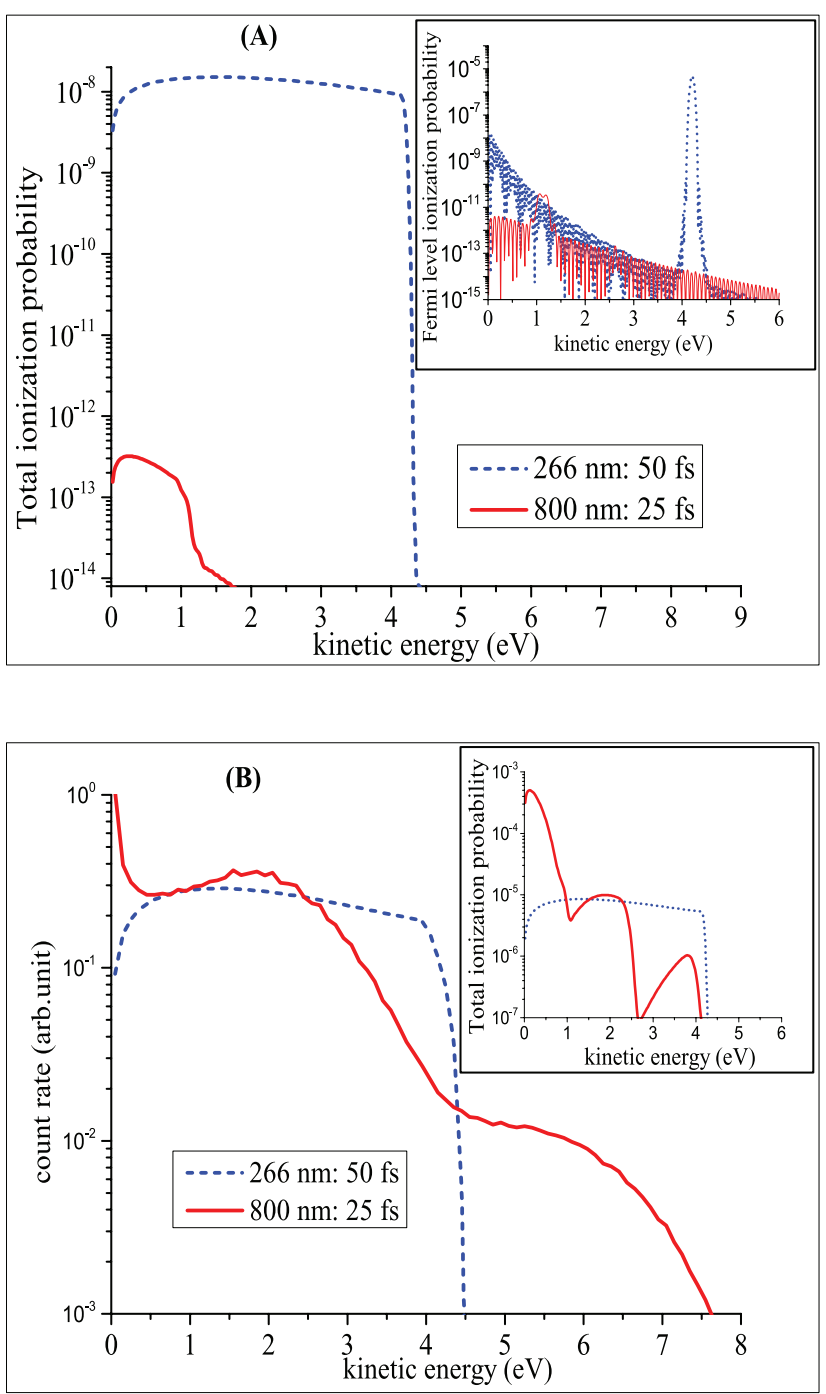

Figure 7. Photoemission spectra as a function of electron ejection energy (in eV) for $800 \mathrm{~nm}, 25 \mathrm{fs}, 1.3 \mathrm{GW} \mathrm{cm}^{-2}$ (full red line) and for $266 \mathrm{~nm}, 50 \mathrm{fs}, 1.3 \mathrm{GW} \mathrm{cm}^{-2}$ (dashed blue line), (A) case of a gold single crystal calculated by means of the Jellium-Volkov method (the curves in the inset correspond to the ionization probability for the same laser parameters but at the Fermi level only); (B) case of a surface with SP (full red line: final spectrum obtained for $800 \mathrm{~nm} \eta=80$ and $\delta=650 \mathrm{~nm}$; dashed blue line: same for $266 \mathrm{~nm}, \eta=20$ and $\delta=25 \mathrm{~nm}$ ). In the inset, the corresponding primary spectra obtained with the JV method in the first step (see text) are displayed.

quite remote from the one that underlies the description of an optical absorption spectrum [51, 52]. Therefore, we focus our attention onto the fact that it is the roughness of the surface of the N-O array target, that triggers the excitation of the SP wave. Hence we do not try to model explicitly the complicated electromagnetic field associated with the SP which is excited on the N-O array target, as has been done in [53]. We rather use the model developed in our previous work on a grating target [27] for a preliminary approach. In this model one first calculates the quantum ionization probabilities in the presence of an external field by means of the Jellium-Volkov method. The external field can be either the laser field or the SP field. After that the final photoelectron spectrum is obtained by taking into account the motion in vacuum of the freed electrons within this external field. We focus our attention here on the tails of the energy spectra. For the N-O array target, these spectra extend to higher energies when the laser wavelength increases from $266 \mathrm{~nm}$ to $800 \mathrm{~nm}$ (see figure 5). For the single crystal, the opposite behaviour is observed (see figure 4).

3.3.1. Case of the gold single cristal. In the case of the single-crystal target, the photons of the impinging laser beam cannot couple to a surface plasmon. Hence the external field in the model is the laser field and the single-crystal target goes into standard laser photo-emission in the low intensity-field of the laser used in the present study: $10^{8}$ to $5 \times 10^{10} \mathrm{~W} \mathrm{~cm}^{-2}$. The quantum ionization probabilities are calculated as a function of emitted electron energy, by means of the JelliumVolkov (JV) method whose details can be found in [27] and [54]. In this perturbative method, whose use is justified by the low intensities we are considering here, the solid metal's electronic structure is described as usually through the jellium model, using the values $E_{\mathrm{F}}=5.5 \mathrm{eV}$ for the Fermi energy and $W=5.1 \mathrm{eV}$ for the work function [47]. This standard approach has been chosen as it is less time-consuming than non-perturbative approaches such as the solution of the TDSE (time dependent Schrödinger equation) [54, 55] or methods based on the more refined TDDFT (time dependent density functional theory).

In figure $7(\mathrm{~A})$ we display the total ionization probabilities obtained in the case of the gold single crystal (i.e. integrated over the whole conduction band) at the two wavelengths of 800 and $266 \mathrm{~nm}$. In the inset we also present the ionization probability corresponding to the Fermi level. These data will be used to analyze the results. The IR curve in the inset presents a peak at $1.1 \mathrm{eV}$, which corresponds to the absorption of four IR photons $(800 \mathrm{~nm}, 1.55 \mathrm{eV})$ in agreement with the photoelectric balance. The UV spectrum in the inset presents a more rapidly decreasing background than the IR one and shows a strong peak at $4.2 \mathrm{eV}$ which corresponds to the emission of electrons through the absorption of two UV photons $(266 \mathrm{~nm}, 4.65 \mathrm{eV})$. As it can be seen in figure 7(A), these peaks are broadened after integration over the conduction band. In the IR case, the integration results in a weak peak extending up to $1.2 \mathrm{eV}$ related to the four photons emission of a narrow $(1.1 \mathrm{eV})$ band of states below the Fermi level. In the UV case, the integration leads to an intense and broad peak (up to $4.4 \mathrm{eV}$ ) resulting from the two UV photons emission of a large $(4.2 \mathrm{eV})$ band of states below the Fermi level. These results are in qualitative agreement with the observed wavelength dependence in the gold single-crystal target. In particular, they provide an explanation for relative energy position of the main peaks. They are also in agreement with the previous discussion of the high-energy tail that is observed when the single crystal is excited by a UV laser pulse with an intensity of $1.3 \mathrm{GW} \mathrm{cm}^{-2}$. At the higher intensity of $32 \mathrm{GW} \mathrm{cm}^{-2}$, the produced electron density is expected to be significantly larger. The result of this is that the electron-electron collisions affect the energy distribution of the ejected electrons. In order to take this point into account, it would be necessary to develop an improved model that takes into account the many-body effects, but this is beyond the scope of the present work. 
3.3.2. Case of surface plasmon excitation. In the $\mathrm{N}-\mathrm{O}$ array target a propagative surface plasmon excitation is present. In the experiments (see figure 5) we observe then also an important extension of the electron spectrum towards higher energies. However it is important to note that in this case the excitation is due to the IR pulse, in sharp contrast with what happens within the single-crystal target. As previously mentioned our experimental results reveal a dependence of the $\mathrm{N}-\mathrm{O}$ array target photoemission on the laser angular incidence that is reminiscent of the data that can be obtained on a grating. In this respect our situation differs markedly from other nano-particle excitation schemes whereby a non-propagative $\mathrm{SP}$ is excited in regime of very high laser intensities $[28,56]$.

In order to understand the behaviour of the N-O array target, we have thus performed calculations using the model of photoemission developed in [27]. This model takes into account the presence of an inhomogeneous surface plasmon electric field that occurs outside the solid and whose intensity is locally stronger than the field of the laser. This approach has been proved to describe fairly well the ponderomotive effects in the presence of SP waves. Its success is rooted in the fact that the electron acceleration is related to the amplitude of the ponderomotive potential rather than to the exact form of the local field when the electron can fully explore the spatial extension of the field. Since the analytical expressions for the electromagnetic field of the surface plasmon waves on the arrays considered are not known, we describe the surface plasmon in the present calculations in the same way as in [27]. For sure, such a preliminary description could be improved upon, e.g. by taking the numerical output fields obtained from standard electromagnetic simulations [57] whereby the shape of our surface is taken into consideration. But such a refinement is beyond the scope of the present work, which merely focuses on the wavelength dependence of the electron energy gain (in the presence of an SP excitation or otherwise) and on the tendencies one may discern in its effects on the photoemission spectra.

In our model, the SP-stimulated electron emission process is treated as a two-step mechanism. The first step considers the electronic transitions from the metallic conduction band towards the solid continuum that are induced by the surface plasmon field. Just like in the single-crystal case it is described by means of the JV method. The corresponding primary spectra are reported in the inset of figure 7(B) for the same laser parameters as those of figure 5, and with an enhancement factor $\eta$ of 80 in the $800 \mathrm{~nm}$ case. Here $\eta=E_{\mathrm{SP}} / E_{0}$, where $E_{\mathrm{SP}}$ and $E_{0}$ are the amplitudes of the Surface Plasmon field and of the laser field, respectively. The choice of this value is consistent with the one obtained in [27] in the IR case. In the UV case we have taken $\eta=20$ as it is known [58] that the enhancement factor is much less important in the UV case than in the IR case. This is in part due to the variations of the real and imaginary parts of the dielectric function of the metal as a function of the wavelength of the impinging photons (from [59] one has: $\epsilon(800 \mathrm{~nm})=-22.7+0.75 i$ and $\epsilon(266 \mathrm{~nm})=-1.2+4.4 i)$. A further effect may be induced by the geometry, i.e. the roughness of the surface resulting from the large but finite size $(\sim 215 \mathrm{~nm})$ of the objects on the surface.
The primary spectra corresponding to the IR and UV cases reported in the inset of figure 7(B), have been obtained by consideration of a surface plasmon field intensity of $5.6 \mathrm{~V} \mathrm{~nm} \mathrm{~nm}^{-1}$ at $800 \mathrm{~nm}$ and $1.4 \mathrm{~V} \mathrm{~nm}^{-1}$ at $266 \mathrm{~nm}$. The IR primary spectrum shows three peaks located around $0.5,2$ and $3.5 \mathrm{eV}$ corresponding to the absorption of four, five and six photons, respectively. For the UV primary spectrum, one observe only one broad peak similar to the one reported in figure 7(A) and related to a two UV photons absorption process. These primary spectra have similar intensities in spite of the fact that the two UV photon absorption process is more probable than the higher order IR ones. This comes from the fact that the enhancement factor is more important in the IR case than in the UV one.

The second step of the model generates the final spectra of figure 7(B). It is based on a classical description of the motion of the freed electrons along the two spatial components of the inhomogeneous oscillating surface plasmon field in the region outside the target surface. During their motion these electrons gain more or less energy. This depends in fact on their initial conditions. The latter are provided by the quantum calculations of the first step in terms of the ejection energy, the emission angle and the instant of release. Note that the electron acceleration, deceleration and re-acceleration which we discussed previously and which contribute to the broadening of the final electron energy distribution towards higher values [15, 17, 49, 50] is also taken into account in the present model. Again, due to the important variations of the dielectric function (of its real part in this case) with respect to the energy of the photon, the decay length $\delta$ of the SP field outside the solid in the IR case is $\delta_{800} \simeq 594 \mathrm{~nm}$ and $\delta_{266} \simeq 25 \mathrm{~nm}$ in the UV one. Therefore, the electrons emitted by the IR pulse are gaining more energy outside the metal than those ejected by the UV pulse, even though both types of pulses convey essentially the same initial energy to the electrons. The reason for the difference is the fact that the enhancement factor and the decay length are greater in the IR case than in the UV case. As can be seen in figure 7(B), this results in a weak shift towards higher energies in the final UV $(266 \mathrm{~nm})$ spectrum with respect to the corresponding primary spectrum, while the shift is large in the IR $(800 \mathrm{~nm})$ case. In the latter case, there is also a high-energy tail related to the five and six photons IR absorption process. This is consistent with the conclusions that can be drawn from the experimental data shown in figure 5 and with the variation of the electron ponderomotive acceleration within the local induced surface plasmon field which varies as $I \lambda^{2}$.

In conclusion, this preliminary model validates our interpretation of the experiments. It demonstrates the variation of the ponderomotive acceleration with the laser wavelength. As previously stated, this model does not take into account the electron-electron interactions inside the emitted bunch. This effect gets growing importance when the number of ejected electrons increases. This explain why the range of kinetic energy obtained in the figure 7(B) is significantly narrower than that shown in the experimental data of figure 5. In order to take this effect into account, it would be necessary to develop an improved model that takes into account the motion of the freed electron cloud outside the solid, but this is beyond the scope of the present work. 

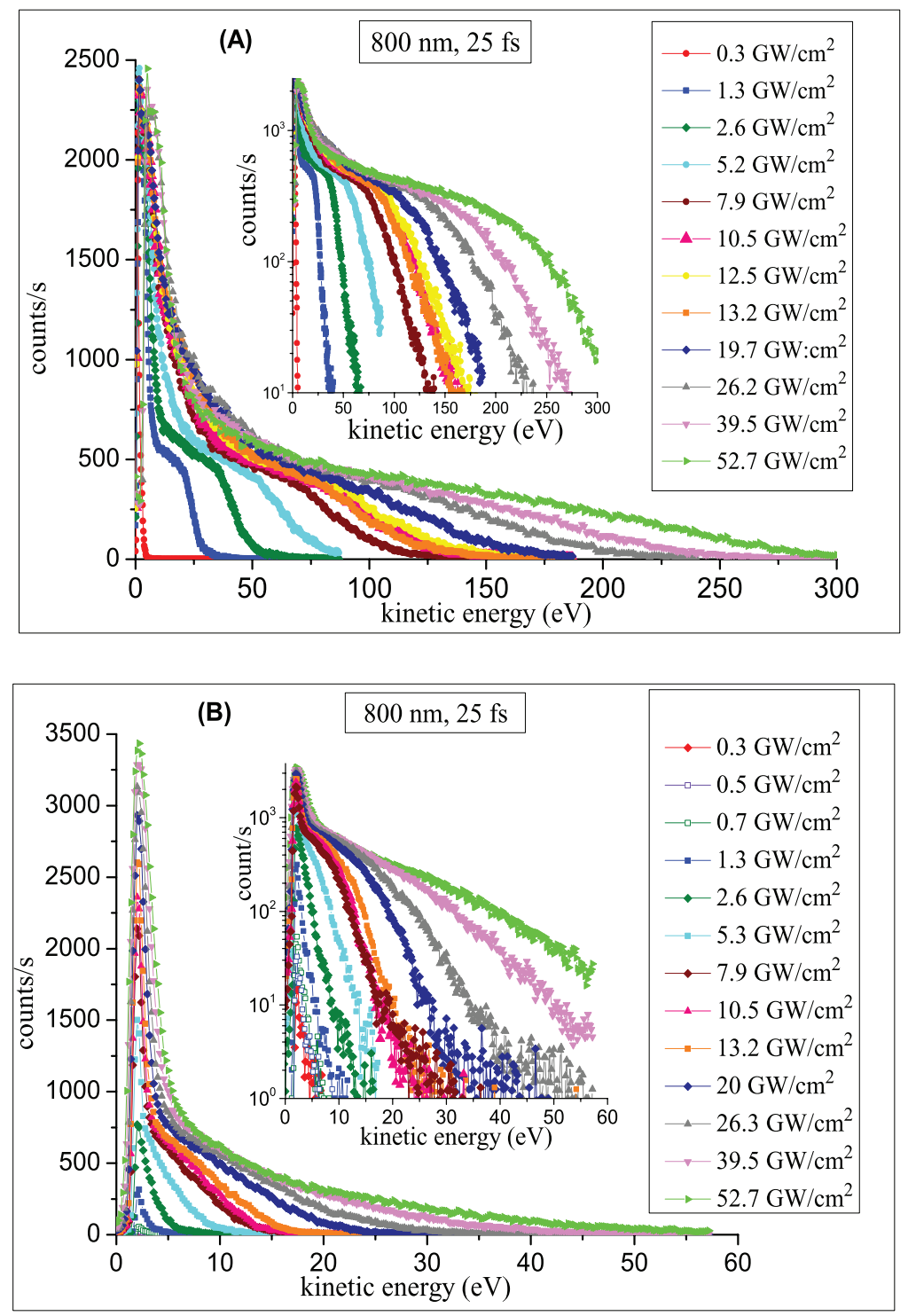

Figure 8. Spectra of photo-emitted electrons from the N-O array target (A) and the gold single-crystal target (B) irradiated with a $800 \mathrm{~nm}$ laser pulse with the laser intensity ranging from: $0.3 \mathrm{GW} \mathrm{cm}^{-2}$ to $53 \mathrm{GW} \mathrm{cm}^{-2}$ (inset: same with a log scaling for the counts s${ }^{-1}$ ). The laser pulse duration is $25 \mathrm{fs}$.

\subsection{Regime of high laser intensities}

As previously shown in section 2.2 (see figure 5), increasing the laser intensity leads to the production of electrons with high kinetic energies in the $\mathrm{N}-\mathrm{O}$ array target. In figure 8(A), we have reported the photo-emitted electron spectra of this target irradiated with a $800 \mathrm{~nm}$ laser pulse of $25 \mathrm{fs}$ pulse duration and a laser intensity ranging from: $0.3 \mathrm{GW} \mathrm{cm}^{-2}$ to 53 $\mathrm{GW} \mathrm{cm}{ }^{-2}$. These laser intensities are well below the damage threshold of the targets.

It is worth noting that electrons with energies as high as $300 \mathrm{eV}$ have been obtained for a laser intensity of only $53 \mathrm{GW} \mathrm{cm}^{-2}$. In the same range of laser intensities, the electrons emitted from the gold single crystal acquire much less kinetic energy as shown by figure $8(\mathrm{~B})$ : at $I=26 \mathrm{GW} \mathrm{cm}^{-2}$, we obtain electrons of $45 \mathrm{eV}$ with the single crystal against $240 \mathrm{eV}$ with the $\mathrm{N}-\mathrm{O}$ array target. It also appears that the higher the intensity is, the smoother the distribution for the high-energy part of the spectra turns out to be. This behaviour is consistent with the behaviour of an equilibrated electron gas, as previously discussed. The slope of the distribution is related to the electron temperature. Indeed, the higher the intensity is, the more efficiently the electrons will be heated by the laser and the higher the temperature will be, thus leading to smoother energy distributions when the laser intensity increases.

To conclude this study of the N-O array targets, we have compared in figure 9 the maximum kinetic energy of the electrons as a function of the laser intensity for the two laser wavelengths of $800 \mathrm{~nm}$ and $266 \mathrm{~nm}$. As observed previously, with the longer wavelength one obtains electrons of higher energies. Moreover, in the range of intensities studied there is no sign whatsoever of a saturation with laser intensity of the maximum energy the electrons can reach when the target is excited at $800 \mathrm{~nm}$, while saturation effects do seem to occur at $266 \mathrm{~nm}$. A fit of these data based on a power dependence provides an exponent of roughly 0.61. Assuming that the electron gas is free, independent and in equilibrium at a temperature 


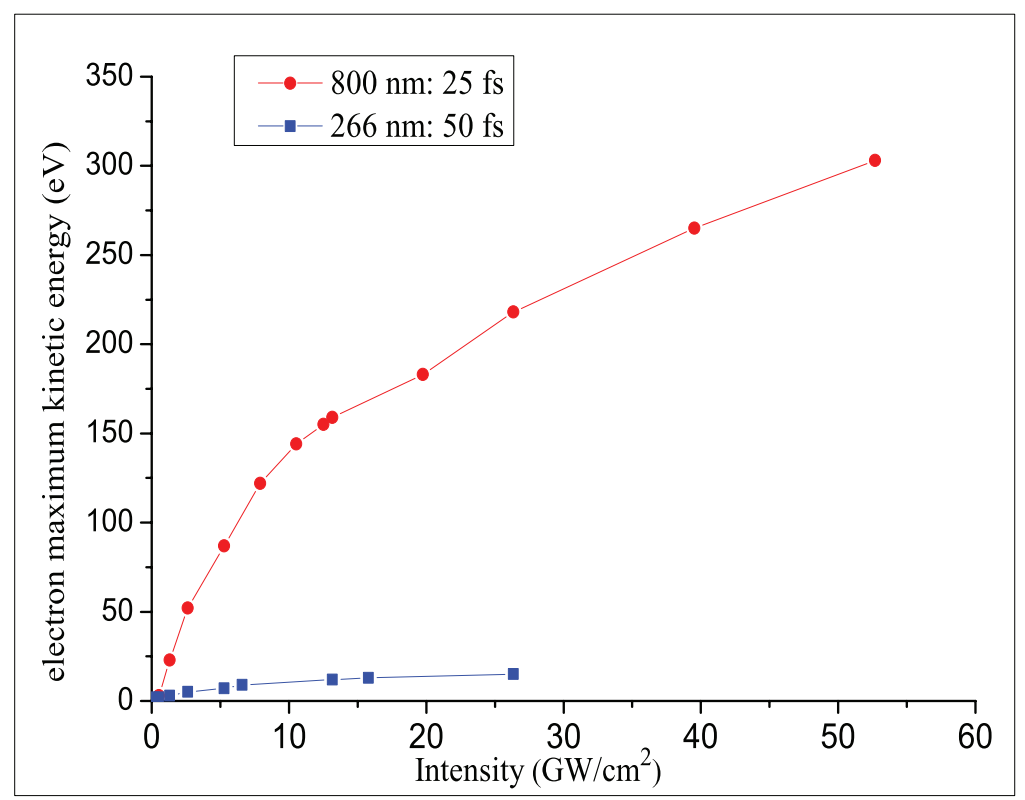

Figure 9. Maximum kinetic energy of the electrons from the $\mathrm{N}-\mathrm{O}$ array target irradiated with a $800 \mathrm{~nm}$ and a $266 \mathrm{~nm}$ laser pulse as a function of the laser intensity.

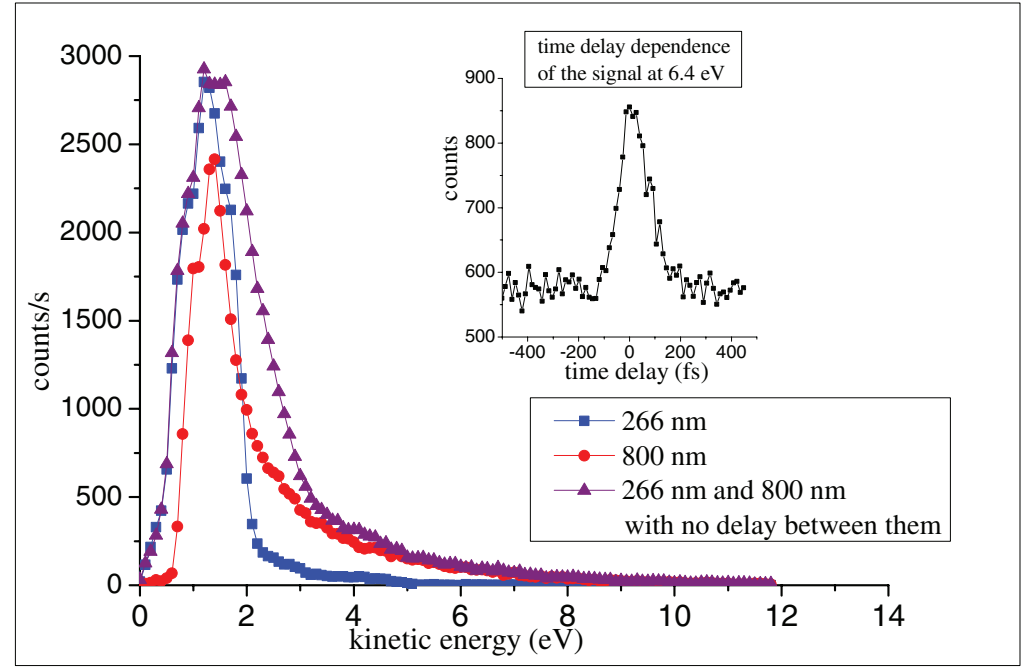

Figure 10. Spectra of photo-emitted electrons in the case of the gold single-crystal sample irradiated with the $800 \mathrm{~nm}$ beam alone at $\sim 5.5 \mathrm{GW} \mathrm{cm}^{-2}$ (red filled circles), with the $266 \mathrm{~nm}$ beam alone at $\sim 0.13 \mathrm{GW} \mathrm{cm}^{-2}$ (blue filled squares) and with both laser beams when there is no-delay between them (violet filled triangles). The inset shows the delay time dependence of the signal at $6.4 \mathrm{eV}$.

significantly smaller than the Fermi temperature, one finds that the maximum energy the electrons can reach lies close to the Fermi energy. This Fermi energy varies as $n_{\mathrm{e}}^{2 / 3}$ with the electron density $n_{\mathrm{e}}$. When the electron density is proportional to the laser intensity, the maximum energy is observed to vary as $I^{0.61}$, and thus as $n_{\mathrm{e}}^{0.61}$. The exponent is close to $2 / 3$, which provides further support to the idea that the aforementioned relaxation processes are part of the mechanism that prevails during the emission.

The previous observations clearly highlight the interest of using longer wavelengths. By tailoring the geometry of the array of the nano-objects and concomitantly the geometry of the SP, one could obtain electron pulses of higher energies. This could open the way to new prospects in plasmon-driven electron sources and lightwave electronics. In particular, this makes this type of target of special interest for processes that involve the emission of hot electrons and high currents.

\subsection{Two-color pump-pump experiments}

Two-color pump-pump experiments have also been performed. The aim of these experiments was to compare the electronic relaxation dynamics that take place in the $\mathrm{N}-\mathrm{O}$ array target when the SP is excited with the dynamics that occur in the gold single-crystal sample. In these experiments a first pump is operated at a wavelength of $800 \mathrm{~nm}$, an intensity of $\sim 5 \mathrm{GW} \mathrm{cm}^{-2}$, and an incidence angle of $45^{\circ}$. It is used to excite the single-crystal target or the SP in the N-O array target. The energy of the photons is less than the threshold for inter-band transition in gold (2.3 eV [45]), such that the pump 

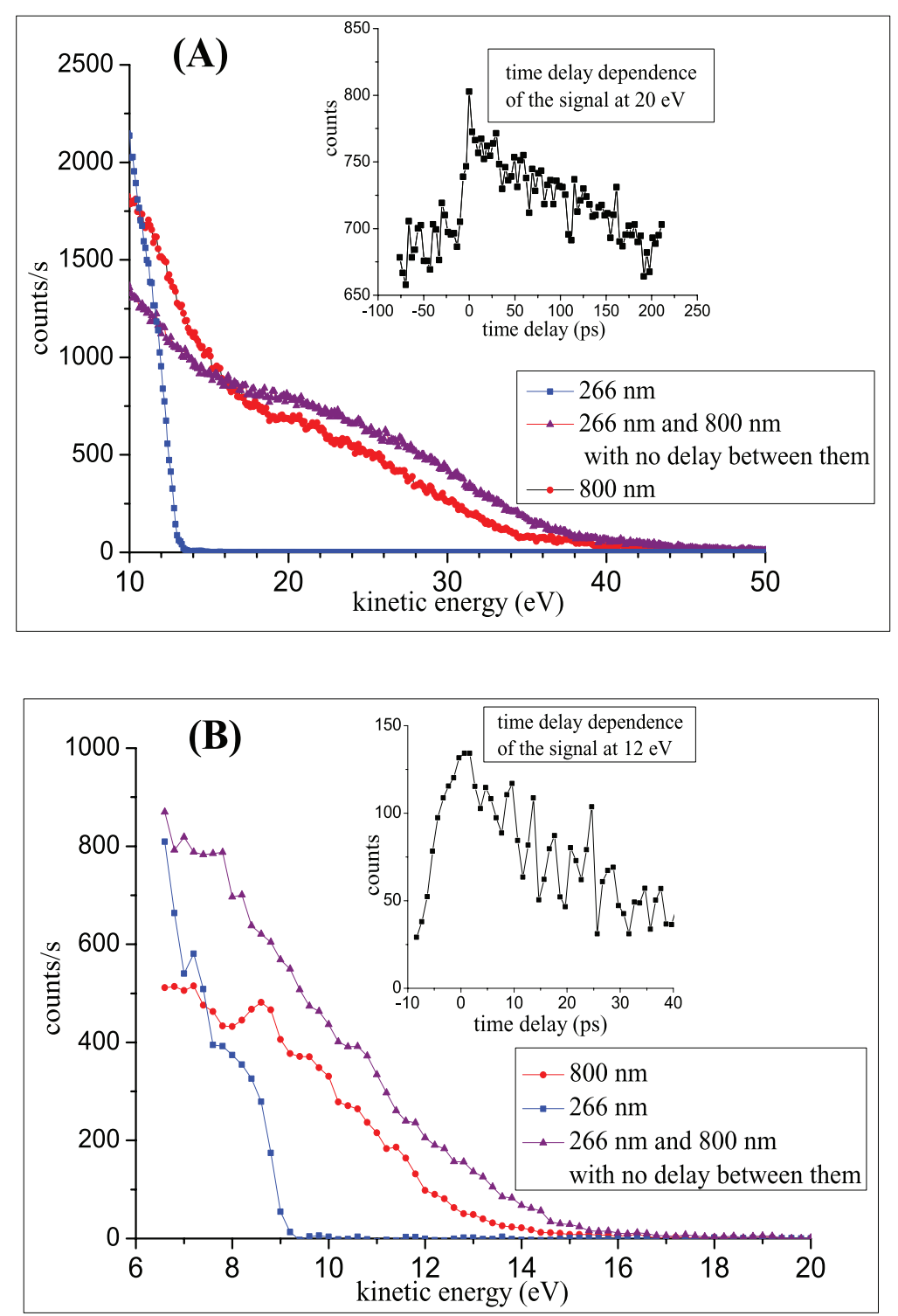

Figure 11. Spectra of photo-emitted electrons: (A) for the NO target $(r \sim 215 \mathrm{~nm})$ irradiated with the $800 \mathrm{~nm}$ beam alone at $\left.\sim 1.8 \mathrm{GW} \mathrm{cm}^{-2}\right)$ (red filled circles), the $266 \mathrm{~nm}$ beam alone at $\sim 1 \mathrm{GW} \mathrm{cm}^{-2}$ (blue filled squares) and with both beams when there is no-delay between the two laser beams (violet filled triangles), and (B) for the NO-100 target $\left(r \sim<100 \mathrm{~nm}\right.$ ) irradiated with the $800 \mathrm{~nm}$ beam alone at $\sim 5 \mathrm{GW} \mathrm{cm}^{-2}$ ) (red filled circles), the $266 \mathrm{~nm}$ beam alone at $\sim 0.1 \mathrm{GW} \mathrm{cm}^{-2}$ (blue filled squares) and with both beams when there is no-delay between them (violet filled triangles). The inset gives the delay time dependence of the signal at $20 \mathrm{eV}(\mathrm{A})$ and $12 \mathrm{eV}(\mathrm{B})$.

creates a transient non-equilibrium electron distribution. The second pump is operated at $266 \mathrm{~nm}$ with a lower intensity than that of the IR pulse. The aim of the set-up is to monitor the changes induced in the sample photoemission as a function of the delay between the two laser beams. The angle of incidence of the $266 \mathrm{~nm}$ laser beam is $30^{\circ}$. This choice is motivated by our desire to stay far out of tune with the SP excitation when we study the N-O array target.

The spectra of the photo-emitted electrons obtained from the gold single-crystal sample are reported in figure 10. It shows the data obtained with: the $800 \mathrm{~nm}$ pump alone (red line), the $266 \mathrm{~nm}$ pump alone (blue line) and both laser beams simultaneously but without any delay between them (magenta line). The reference spectra $(800 \mathrm{~nm}$ alone and $266 \mathrm{~nm}$ alone) show the undisturbed electron energy distributions obtained with each individual pump beam. The spectrum obtained with the two-color beam in zero delay is also reported and exhibits a tail above $6 \mathrm{eV}$. This tail accounts for the changes induced within the gold single crystal. In the inset of figure 10, the evolution of the signal (at $6.4 \mathrm{eV}$ ) as a function of the time delay between the two pulses is shown. A fit of the decrease of the signal intensity with an exponential function yields a characteristic time of $\tau=78 \mathrm{fs}$ ( $\pm 5 \mathrm{fs}$ ). This value is in agreement with previous values reported for gold $[60,61]$ and accounts for the fast electron-electron relaxation dynamics towards a quasi-equilibrium distribution. This takes place on a timescale much shorter than that of a cooling mechanism based on an energy transfer to the lattice through electron-phonon collisions [62, 63].

As figure 11(A) shows, the time dependence of the signal obtained with the $\mathrm{N}-\mathrm{O}$ array target is quite different from that obtained with the single-crystal sample. On rough surfaces or 
in large nano-objects (that are deposited on some support), the relaxation dynamics of an electron distribution that has not yet reached its equilibrium can be influenced by thermal effects. These can be due to the finite size of the objects or to the shape of the roughness. Indeed, in the case of the gold single-crystal the interaction area is very large (in the $\mathrm{mm}$ scale). The time scales on which heat diffusion (which is here significant) and the evolution of the electron and lattice temperatures towards equilibrium take place are in the present experiment much longer than the time delay between the two pulses. There is thus no evolution of the photoemission signal to be expected in response to a variation of the temperature. The rough surface under study here, is constituted of finite-size objects that may increase the heat transfer significantly. This will lead to a fast decrease of the temperature, possibly on a timescale of the same order of magnitude as that of the delay time between the two laser pulses. The fastest dynamics are dominated by electron-electron and electron-phonon relaxation in the objects, which take place on a timescale of the order of hundred fs and some ps, respectively. After this intrinsic relaxation has taken place, the heat diffusion within the substrate is expected to take place on a longer timescale, which is of the order of hundreds of ps. An influence on the temporal evolution of the photo-emission signal is thus expected [42]. The two following possible mechanisms can be suggested. First, since the electrons are initially distributed according to the Fermi-Dirac statistics [27], the higher the temperature is, the higher the initial energy of electrons will be. This modification in the initial conditions for the energy should thus have an influence on the spectrum of the ejected electrons after their interaction with the laser has taken place. Second, just like in the one-color case, one may assume that heating mechanisms are also at work the bulk. They are due to the aforementioned electron-phonon-photon collisions, whose efficiency depends on the phonon population [48]. Since the latter evolves with the temperature of the material, such a mechanism is expected to have an influence on the final energy distribution of the ejected electrons. The inset of figure 11(A) shows the dependence of the signal on the delay time for an electron energy of $20 \mathrm{eV}$. As expected, its deconvolution now exhibits two characteristic decay times, $\tau_{1} \sim 830 \mathrm{fs}( \pm 50 \mathrm{fs})$ and $\tau_{2} \sim 117 \mathrm{ps}( \pm 5 \mathrm{ps})$. They are both much longer than the decay time measured with the gold single-crystal.

In order to study the influence of the finite size of the objects, we have complemented this study with pump-pump experiments on a new target with nano-objects of smaller size (radius $<100 \mathrm{~nm}$ ). This target will hereafter be called the NO-100 target. The inset of figure 11(B) shows the dependence of the signal from this target on the delay time for an electron energy of $12 \mathrm{eV}$. Its deconvolution yields two characteristic decay times, viz. $\tau_{1}=1 \mathrm{ps}( \pm 50 \mathrm{fs})$ and $\tau_{2}=36 \mathrm{ps}$ ( $\pm 2 \mathrm{ps}$ ). The first decay time is due to the electron-phonon collisions and its value is fairly close to the value found in the $\mathrm{N}-\mathrm{O}$ array target where the radius of the nano-objects is larger. The observation that the characteristic time $\tau_{1}$ is nearly constant is consistent with the fact that electron-phonon collisions are local processes that do not depend on the size of the region where they take place. Size effects can only be expected when the size of the nano-particles is very small, i.e. in the range of a few nanometers [5]. Indeed for such small sizes, the phonon properties may change because the periodic boundary conditions are no longer valid. The electron-phonon interactions may therefore change as well. The second characteristic time $\tau_{2}$ accounts for the heat transfer from the nano-objects to the substrate. Its value in the $\mathrm{N}-\mathrm{O}$ array target with a smaller radius decreases to $\sim 36 \mathrm{ps}$ ( $\pm 2 \mathrm{ps}$ ). This observation is consistent with the mechanism proposed to take into account the influence of the heat transfer and the temporal evolution of the lattice temperature on the photoemission signal. Indeed, the characteristic length $l_{\mathrm{c}}$ and time $t_{\mathrm{c}}$ of a diffusion processes are related according to the equation $l_{\mathrm{c}}=2 \sqrt{D t_{\mathrm{c}}}$ where $D$ is the diffusion coefficient. Consequently, the smaller the radius of the object is, the shorter the relaxation time will be, and this is what we observe. In addition, the diffusion coefficient for bulk gold is $D=1.27 \times 10^{-4} \mathrm{~m}^{2} \mathrm{~s}^{-1}$, such that lengths in the range of hundreds of nm correspond to timescales in the range of the hundreds of ps. These values are consistent with the present observations. Moreover, the square root dependence in the equation $l_{\mathrm{c}}=2 \sqrt{D t_{\mathrm{c}}}$ is also satisfied, i.e. the ratio of the sizes of the nano-objects is roughly the square root of the ratio of the characteristic times $\tau_{2}$. These considerations support thus the physical mechanism we have proposed to explain the influence of the lattice temperature on the photo-emission process.

\section{Conclusion}

We have presented a study in the short-(laser)pulse regime of the photo-emission properties of metallic regular 2D lattices in the presence of a surface plasmon excitation. The lattice consists of gold nano-objects. We have compared this photoemission with the photo-emission of a gold single crystal. The dependence of the emission on the laser intensity and wavelength has been addressed. The interpretations are supported by a model for the photoemission in the presence of surface plasmons.

We have shown that the use of a gold N-O array target, where the objects are well organized in an 2D array, permits the laser to excite a surface plasmon and modifies the emission of the gold sample drastically. The yield of the electron current emitted from the N-O array target is enhanced by at least a factor of 50 with respect to the single-crystal sample. In these $\mathrm{N}-\mathrm{O}$ array targets one observes also higher kinetic energies than can be expected based on the photoelectric balance. In addition, we have exhibited for the first time, the wavelength dependence of the photoemission from the $\mathrm{N}-\mathrm{O}$ array target when the laser excites a surface plasmon. These results can be well interpreted by means of a model that describes the SP-stimulated electron emission process. A correlation has been established with the variation of the electron ponderomotive acceleration in the local induced surface plasmon field, which varies as $I \lambda^{2}$. Finally, we demonstrate that with increasing laser intensities, we can obtain electrons with kinetic energies as high as $\sim 300 \mathrm{eV}$ at $50 \mathrm{GW} \mathrm{cm}^{-2}$ from the N-O array target. Such a high energy value cannot be 
reached with the gold single crystal under similar irradiation conditions.

We have complemented this single-wavelength study with two-color pump-pump experiments performed in order to investigate the different types of electronic relaxation dynamics that prevail in the $\mathrm{N}-\mathrm{O}$ array target and the gold single-crystal sample. The relaxation dynamics in the $\mathrm{N}-\mathrm{O}$ array target are found to be much slower than in the single crystal. Two timescales for the relaxation are observed in the N-O array target. They depend on the size of the nano-objects and have been attributed to the influence of macroscopic effects such as the heat transfer. This work highlights the favorable role played by the electron ponderomotive acceleration in the local induced surface plasmon field. It opens new possibilities for tailoring the geometry of targets with nano-objects arranged in a network and for optimizing the laser excitation parameter to generate plasmonic electron sources.

\section{Acknowledgments}

This work was supported partially by the Czech Science Foundation grants No. P205/13/20110S and P108/12/G108 and the University of Ostrava project No. IRP201553. GD thanks B Chimier and L Barilleau for fruitful discussions.

\section{References}

[1] Kreibig U and Vollmer M 1995 Optical Properties of Metal Clusters (Berlin: Springer)

[2] Vasa P, Ropers C, Pomraenke R and Lienau C 2009 Laser Photonics Rev. 3483

[3] Mie G 1908 Ann. Phys. 25377

[4] Klar T, Perner M, Grosse S, von Plessen G, Spirkl W and Feldmann J 1998 Phys. Rev. Lett. 804249

[5] Shahbazyan T V, Perakis I E and Bigot J Y 1998 Phys. Rev. Lett. 813120

[6] Varin C, Peltz C, Brabec T and Fennel T 2012 Phys. Rev. Lett. 108175007

[7] Ciraci C, Hill R T, Mock J J, Urzhumov Y, FernandezDomínguez A I, Maier S A, Pendry J B, Chilkoti A and Smith D R 2012 Science 3371072

[8] Raza S, Bozhevolnyi S I, Wubs M and Mortensen N A 2015 J. Phys.: Condens. Matter 27183204

[9] Penn D R and Rendell R W 1981 Phys. Rev. Lett 471067

[10] Monchicourt P, Raynaud M, Saringar H and Kupersztych J 1997 J. Phys.: Condens. Matter 95765

[11] Lehmann J, Merschdorf M, Pfeiffer W, Thon A, Voll S and Gerber G 2000 Phys. Rev. Lett. 852921

[12] Kim S, Jin J, Kim Y J, Park I Y, Kim Y and Kim S W 2008 Nature $\mathbf{4 3 5} 757$

[13] Baida H, Mongin D, Christofilos D, Bachelier G, Crut A, Maioli P, Del Fatti N and Vallée F 2011 Phys. Rev. Lett. 107057402

[14] Bormann R, Gulde M, Weismann A, Yalunin S V and Ropers C 2010 Phys. Rev. Lett. 105147601

[15] Herink G, Solli D R, Gulde M and Ropers C 2012 Nature 483190

[16] Nagel P M, Robison J S, Hartenck B D, Pfeifer T, Abel M J, Prell J S, Neumark D M, Kaindl R A and Leone S R 2013 Chem. Phys. 414106

[17] Dombi P, Hörl A, Rácz P, Márton I, Trüger A, Krenn J R and Hohenester U 2013 Nano Lett. 13674
[18] Kupersztych J, Monchicourt P and Raynaud M 2001 Phys. Rev. Lett. 865180

[19] Zawadzka J et al 2001 Appl. Phys. Lett. 792130

[20] Irvine S E, Dechant A and Elezzabi A Y 2004 Phys. Rev. Lett. 93184801

[21] Irvine S E and Elezzabi A Y 2006 Phys. Rev. A 73013815

[22] Welsh G H, Hunt N T and Wynne K 2007 Phys. Rev. Lett. 98026803

[23] Dombi P and Rácz P 2008 Opt. Express 162887

[24] Dombi P 2009 Adv. Imaging Electron Phys. 1581

[25] Sussmann F and Kling M F 2011 Phys. Rev. B 84121406

[26] Rácz P and Dombi P 2011 Phys. Rev. A 84063844

[27] Jouin H, Raynaud M, Duchateau G, Geoffroy G, Sadou A and Martin P 2014 Phys. Rev. B 89195136

[28] Zherebtsov S et al 2011 Nat. Phys. 7656

[29] Foldi P, Marton I, Nemet N, Ayadi V and Dombi P 2015 Appl. Phys. Lett. 106013111

[30] Liu M, Guyot-Sionnest P, Lee T-W and Gray S K 2007 Phys. Rev. B 76235428

[31] Chu P and Mills D L 2007 Phys. Rev. Lett. 99127401

[32] Chu Y, Schonbrun E, Yang T and Crozier K B 2008 Appl. Phys. Lett. 93181108

[33] Kravets V G, Schedin F and Grigorenko A N 2008 Phys. Rev. Lett. 101087403

[34] Conley G M, Burresi M, Pratesi F, Vynck K and Wiersma D S 2014 Phys. Rev. Lett. 112143901

[35] Meinzer N, Barnes W and Hooper I R 2014 Nat. Photon. 8889

[36] Lamprecht B, Schider G, Lechner R T, Ditlbacher H, Krenn J R, Leitner A and Assenegg F R 2000 Phys. Rev. Lett. 844721

[37] Hicks E M, Zou S, Schatz G C, Spears K G, van Duyne R P, Gunnarsson L, Rindzevicius T, Kasemo B and Kall M 2005 Nano Lett. 51065

[38] Auguiè B and Barnes W L 2008 Phys. Rev. Lett. 101143902

[39] Sung J, Hicks E M, Van Duyne R P and Spears K G 2008 J. Phys. Chem. C 1124091

[40] Raether H 1988 Surface Plasmon on Smooth and Rought Surfaces and on Gratings (Springer Tracts in Modern Physics vol 111) (Berlin: Springer)

[41] Kretschmann E and Reather H 1968 Z. Nat.forsch. A 232135

[42] Pfeiffer W, Kennerknecht C and Merschdorf M 2004 Appl. Phys. A 781011

[43] Ulstrup S et al 2015 J. Phys.: Condens. Matter 27164206

[44] Kosiorek A, Kandulski W, Chudzinski P, Kempa K and Giersig M 2004 Nano Lett. 4 1359-63

[45] Cooper B R, Ehrenreich H and Philipp H R 1965 Phys. Rev. 138494

[46] Petite G, Agostini P, Trainham R, Mevel E and Martin P 1992 Phys. Rev. B 4512210

[47] Michaelson H B 1977 J. Appl. Phys. 484729

[48] Rethfeld B, Kaiser A, Vicanek M and Simon G 2002 Phys. Rev. B 65214303

[49] Kupersztych J, Raynaud M and Riconda C 2004 Phys. Plasmas 111669

[50] Riconda C, Raynaud M, Vialis T and Grech M 2015 Phys. Plasmas 22073103

[51] Yannopapas V 2008 J. Phys.: Condens. Matter 20325211

[52] Persechini L, Verre R, McAlinden N, Wang J J, Ranjan M, Facsko S, Shvets I V and McGilp J F 2014 J. Phys.: Condens. Matter 26145302

[53] Negre C F A, Perassi E M, Coronado E A and Sanchez C G 2013 J. Phys.: Condens. Matter 25125304

[54] Faraggi M N, Gravielle M S and Mitnik D M 2007 Phys. Rev. A 76012903

[55] Lemell C, Tong X-M, Krausz F and Burgdörfer J 2003 Phys. Rev. Lett. 90076403

[56] Fennel Th, Döppner T, Passig J, Schaal Ch, Tiggesbäumker J and Meiwes-Broer K H 2007 Phys. Rev. Lett. 98143401 
[57] Oskooi A F, Roundy D, Ibanescu M, Bermel P, Joannopoulos J D and Johnson S G 2010 Comput. Phys. Commun. 181687

Hohenester U and Trugler A 2012 Comput. Phys. Commun. 183370

[58] Weber W H and Ford G W 1981 Opt. Lett. 6122

[59] Lide D R 2003 Handbook of Chemistry and Physics 84th edn (New York: CRC Press)
[60] Cao J, Gao Y, Elsayed-Ali H E, Miller R J D and Mantel D A 1998 Phys. Rev. B 5810948

[61] Aeschlimann M, Bauer M, Pawlik S, Knorre R, Bouzerar G and Bennemann K H 2000 Appl. Phys. A 71485

[62] Schoenlein R W, Lin W Z, Fujimoto J G and Eesley G L 1987 Phys. Rev. Lett. 581680

[63] Fann W S, Storz R, Tom H W K and Bokor J 1992 Phys. Rev. Lett. 682834 